The Low Surface Brightness Universe, IAU Col. 171

ASP Conference Series, Vol. 170, 1999

J. I. Davies, C. Impey and S. Phillipps, eds.

\title{
Dwarf Galaxies in Nearby Groups
}

\author{
T. Bremnes and B. Binggeli \\ Astronomical Institute, University of Basel, Switzerland \\ P. Prugniel
}

Observatoire de Lyon, France

\begin{abstract}
We present preliminary results from two observing campaigns where global photometric data for most dwarf galaxies in the M81 and M101 groups as well as some field dwarfs were obtained. The galaxies in the denser M81 group are more often of dwarf elliptical type and are redder and fainter than those of the M101 group and surrounding field, which are mostly of the dwarf irregular types. But both types follow the same total magnitude - central surface brightness relation, so there might be an evolutionary connection between the two classes.
\end{abstract}

\section{Introduction}

Our work is aimed at filling the gap of data and knowledge between LG and cluster dwarfs. We have until now determined global photometric properties of members of the M81 and M101 groups (Bremnes et al. 1998a, Lesaffre et al. 1998, Bremnes et al. 1998b) as well as possible M101 group members and nearby field galaxies. These observations are part of a long term project aimed at doing systematic imaging of dwarf galaxies in nearby groups and the general field, based on the "10 Mpc Catalogue" of galaxies by Kraan-Korteweg and Tammann (1979), updated by Schmidt and Boller (1992). Our goal is to compare these galaxies with existing data on the dwarf galaxy populations of the LG and clusters such as Virgo and Fornax. Total magnitudes, effective radii, effective surface brightnesses and galaxy diameters at various isophotal levels have been determined in the Cousins $B$ and $R$ bands as well as best-fitting exponential parameters and colour gradients.

\section{The ABC of dEs dIs BCDs ...}

There is evidence pointing towards the existence of an evolutionary link between dwarf irregulars, BCDs and dwarf ellipticals (Evans et al. 1990, Patterson \& Thuan 1996, Moore et al. 1998). Their structural parameters are quite similar. They form continuous sequences in different parameter spaces, like for example the central surface brightness - absolute magnitude plane. The existence of a density - morphology (Dressler 1980, Binggeli et al. 1987, Ferguson and Sandage 1988, Binggeli et al. 1990) or density - dwarf population (Phillipps et al. 1998) 
relation also points towards the possibility of an evolutionary link between the different types. Dwarf ellipticals are often associated with large galaxies, dense groups and clusters, whereas the irregulars are mostly present in the field and loose groups. There is also a clear difference in the distribution of the different galaxy types in the CM diagram: the early galaxy types populate the fainter and redder part, whereas the late-type galaxies are bluer and brighter. Note that in dense clusters, the trend is opposite, the fainter dEs being bluer and fainter than the brighter dEs (Secker et al. 1997), but see also Evans et al. (1990). The general picture could be one where the dEs are the remnants of spirals or dIs which have been "harassed" by their cluster/group environment (Moore et al. 1998). The M81 group dwarfs are on average redder and fainter than those of the less dense M101 group and the field dwarfs, which are still bluer and brighter, see Fig. 1. This is to be expected if for example the dEs were dIs that were "harassed" by their environment to produce stars earlier and with a larger SFR, whereas the field dIs simply float around, forming stars in a "quiescent" manner over a much longer timescale (Van Zee et al. 1997).

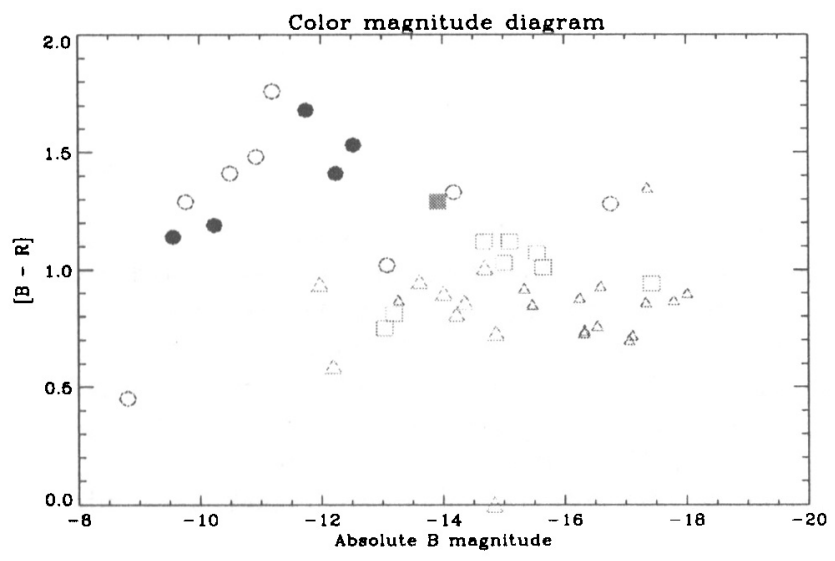

Figure 1. CM diagram for dwarf galaxies in the M81 (circles) and M101 (squares) groups as well as field dwarfs (large triangles) in the projected vicinity of M101 and "quiescent" dwarf irregulars (small triangles) from Van Zee et al. (1997). Filled symbols represent early-type galaxies, open ones late-type galaxies.

\section{References}

Binggeli, B., Tammann, G. A. and Sandage, A. 1987, AJ, 94, 251

Binggeli B., Tarenghi, M. and Sandage, A. 1990, A\&A, 228, 42

Bremnes, T., Binggeli, B. and Prugniel, P. 1998a, A\&AS, 129, 313

Bremnes, T., Binggeli, B. and Prugniel, P. 1998b, A\&AS, in preparation

Dressler, A. 1980, ApJ, 236,351 
Evans, R., Davies, J. I. and Phillipps, S. 1990, MNRAS, 245, 164

Ferguson, H. C. and Sandage, A. 1988, AJ, 96, 1520

Kraan-Korteweg, R. C. and Tammann, G. A. 1979, AN, 300, 181

Lesaffre, P., Prugniel, P., Binggeli, B. and Bremnes, T. 1998, A\&AS, in preparation

Moore, B., Lake, G. and Katz, N. 1998, ApJ, 495, 139

Patterson, R. J. and Thuan, T. X. 1996, ApJS, 107, 103

Phillipps, S., Driver, S. P., Couch, W. J. and Smith, R. M. 1998, ApJ, 498, 119

Schmidt, K. H. and Boller, T. 1992, AN, 313, 189

Secker, J., Harris, W. E. and Plummer, J. D. 1997, PASP, 109, 1377

Van Zee, L., Haynes, M. P. and Salzer, J. J. 1997, AJ, 114, 2479 